\title{
Mediation effects of social support on relationships of perceived environment and self-efficacy with school-based physical activity: A structural equation model tailored for Japanese adolescent girls
}

\author{
$\mathrm{Li} \mathrm{He}^{1^{*}}$, Kaori Ishii ${ }^{2}$, Ai Shibata ${ }^{2}$, Minoru Adachi ${ }^{3}$, Keiko Nonoue $^{4,5}$, Koichiro Oka $^{2}$ \\ ${ }^{1}$ Graduate School of Sport Sciences, Waseda University, Tokorozawa, Japan; ${ }^{*}$ Corresponding Author: meethl@moegi.waseda.jp \\ ${ }^{2}$ Faculty of Sport Sciences, Waseda University, Tokorozawa, Japan \\ ${ }^{3}$ Graduate School of Education, Okayama University, Okayama, Japan \\ ${ }^{4}$ Sonan Junior High School, Okayama, Japan \\ ${ }^{5}$ Waseda Institute for Sport Sciences, Tokorozawa, Japan
}

Received 5 December 2012; revised 7 January 2013; accepted 15 January 2013

\begin{abstract}
Background: Identifying correlates of physical activity that can be targeted as potential mediators is important for developing interventions to promote physical activity in adolescent girls. However, the mediated effects of multilevel correlates of physical activity remain poorly understood. Therefore, the present study aimed to examine direct and mediated effects of personal, social and perceived school physical environmental factors on school-based physical activity of Japanese adolescent girls. Methods: In this cross-sectional survey of the Japanese adolescent lifestyles, 344 junior high school girls were invited to complete self-report meas-

directly affected physical activity at school; perceived safety, facilities and self-efficacy exhibited indirect effects on physical activity through family or friend support. However, there were no significant associations between equipment and after-school-hours physical activity. Regardless of contexts, BMI had neither direct nor indirect effects on physical activity. Conclusion: Social support from family and friends was identified as factors mediating the effects of perceived environment and self-efficacy on school-based physical activity among Japanese adolescent girls. This finding encourages the future development of effective interventions to promote physical activity through family and friend support in the future.
\end{abstract} ures of age, grade, weight, height, self-efficacy, social support (family, friends and teachers), perceived school physical environment (equipment, facilities and safety) and physical activity at school (min per week during lunch time and after-school hours). Structural equation modeling analysis controlling for age was performed to examine the effects of body mass index (BMI), self-efficacy, social support and school physical environmental variables on lunchtime and after-hours physical activity. Results: The final structural model demonstrated an acceptable fit for each context-specific physical activity. During lunch recess, perceived equipment and friend support exhibited direct effects on physical activity; perceived facilities, safety, and selfefficacy were indirectly associated with physical activity through friend support. During afterschool hours, both family and friend support
Keywords: Public Health; Physical Activity; Adolescents; Correlates; Ecological Model; Mediator

\section{INTRODUCTION}

Regular physical activity during adolescence has been well demonstrated to have short- and long-term benefits for health [1,2]. However, the majority of adolescents are not physically active. Throughout the world, participation in physical activity declines dramatically from adolescence, particularly in girls [3]. In Japan, girls are less active than boys at all ages. According to the latest national investigation, about $39 \%$ of adolescent girls (vs. $16 \%$ of adolescent boys) do not engage in $60 \mathrm{~min}$ of physical activity daily [4]. Considering that physical activity interventions should be designed for specific atrisk groups, adolescent girls are one such group, which 
underscores the interest in developing successful interventions to promote their physical activity.

Such interventions require identifying modifiable correlates of physical activity that can be targeted as potential mediators [5]. However, most previous studies focused on the direct effects of correlates. There is little research investigating indirect effects of factors on physical activity.

From the social ecological perspective, physical activity behavior is determined by an interaction of multilevel influences across personal, social and environmental factors, although previous studies mainly examine psychological and/or social environmental factors [6-12]. Few studies have examined physical environmental influences on physical activity, which are recognized as having long-term effects on population-based health behaviors. Even fewer studies have examined both the direct and indirect effects of multilevel factors on physical activity behavior [13-15].

Moreover, such ecological approach suggests that examined behaviors should be specific to the relevant environment (i.e., the school physical environment is relevant to school-based physical activity). However, existing studies of environmental influences on adolescent physical activity behavior have focused on overall physical activity level without distinguishing physical activity in different contexts (i.e., in certain places and at certain times) [16]. Currently, no studies have reported direct and indirect effects of correlates on context-specific physical activity among adolescents, although a comprehensive understanding of how environmental and personal factors interact to influence individuals' context-specific physical activity behavior is necessary for the development of effective interventions and health policies.

For children and adolescents, schools are important settings for physical activity. Students spend much of their day at school and have many potential opportunities for daily activity there, such as physical education classes, break times (including lunch recess) and after-school hours. Physical education classes alone do not provide them with enough opportunity to meet recommended amounts of physical activity [17]. One review suggested that involvement in physical activity during non-curricular school time contributes $5 \%-40 \%$ of the recommended daily 60 minutes of physical activity [18]. It is therefore important to explore the direct and indirect effects of personal, social and school environmental factors that may influence such non-curricular physical activity at school.

As a particularity of social context among Japanese adolescent girls, the strong desire to thinness or underweight can be listed. Contrary to other developed countries in which increase of obesity are major problems, the prevalence of underweight increased among them [1922]. Society's equating thinness with beauty and attracttiveness in women can be one of reason for this [23]. Thus, contribution of body weight on the relationship between multilevel factors and physical activity may be different between Japanese adolescents and those in other western countries.

On the other hand, increase in autonomy and peer influence is one of relatively common characteristics in adolescence regardless of nationality. Especially, gender influences on these become more pronounced [24]. Compared with boys, adolescent girls, with the earlier development of puberty and self-concept, has been found to be more concerned about the self-appearance, popularity in the class, the evaluation from other classmates and teachers [25]. Thus, even in same school environments, the pattern and direction in relationship between factors and physical activity would be difference between genders. Therefore, further understanding how the multilevel factors interact with each other to develop the tailored intervention strategies to promote physical activity among adolescent girls.

In sum, the present study expanded current literature by examining not only the direct but also the indirect effects of all the selected individual, social and environmental factors with school-based physical activity at different times of days in Japanese adolescent girls.

\section{METHODS}

\subsection{Participants and Data Collection}

Data for the present study were collected from a crosssectional survey of the Japanese adolescent lifestyle. Data collection was conducted between Oct. and Dec. 2010. A total of 761 public junior high school students (aged 12 - 15 years old) in Japan participated in this survey, including 344 girls. All participants were invited to complete a self-report questionnaire individually during class time. Information on demographic such as age, gender and grade were collected with this questionnaire. Informed consent was obtained from all participants, their guardian and the school. Participation was voluntary, and confidentiality was ensured. The study protocol was approved by the Research Ethics Committee of Waseda University.

\subsection{Measures}

Measures of weight and height were collected through a height and weight measuring scale. Body mass index (BMI) was calculated. Participants were grouped into underweight, normal weight, overweight and obesity by BMI ranges specific for age and gender using Centers for Disease Control and Prevention criteria [26,27]. 
Participants were required to report the frequency (day/week) and duration (min/day) of physical activity at school during lunch recess and after hours in a usual week. For analysis, minutes per week were calculated for each setting (lunchtime physical activity and after-school physical activity) [28].

Based on a previous instrument [29], 10 items were used to assess three factors of school physical environment. The three factors were 1) "equipment" (3 items), examining the accessibility or usability of physical equipment (e.g., There is enough equipment for activities at school); 2) "facilities" (4 items), measuring the accessibility or usability of physical activity facilities (e.g., The school grounds are big enough for activities); and 3) "safety" (3 items), investigating perceived safety of physical activity equipment and facilities (e.g., It is safe to engage in physical activity on the grounds and in the gym at school). All items were rated on a four-point scale from 1) strongly disagree to 4) strongly agree. The factorial reliability (equipment: Cronbach $\alpha=0.71$; facilities: Cronbach $\alpha=0.75$; and safety: Cronbach $\alpha=$ 0.83 ) of this scale was confirmed by respondents.

In terms of social support for physical activity, participants were asked to rate support from three sources on a four-point scale from 1) not supportive at all to 4) strongly supportive for the following question: "How do you rate support for engaging in physical activity from 1) family; 2) teachers at school and 3) friends at school?".

The measure of self-efficacy related to physical activity (i.e., belief in one's ability to be active relative to peers [30]) contained 1 item with responses ranging from 1) strongly disagree to 4) strongly agree. The statement was "I am able to do physical activities/exercises/sports better than my friends."

\subsection{Data Analysis}

Only girls with complete data $(\mathrm{N}=280)$ were included in the analyses. Means and standard deviations (SD) were then calculated through descriptive statistics using SPSS18.0 software. The size of the final sample was adequate to estimate the models [31]. Finally, structural equation modeling (SEM) analysis with maximum likelihood estimation in Amos 17.0 was performed to test the fit of proposed models.

The original proposed model that led to a good model fit of the final model is described below. The measurement model included (a) three latent variables of physical environment: equipment (3 indicators), facilities (4 indicators) and safety (3 indicators); (b) relations between latent variables and their indicators and (c) correlations between the three latent environmental factors. The structural model included (a) paths from perceived physical equipment, facilities and safety and BMI to perceived self-efficacy and self-reported physical activity; (b) path from self-efficacy to each source of social support and (c) paths from self-efficacy and three sources of social support to physical activity.

Model fit was assessed using the goodness-of-fit index (GFI), adjusted goodness-of-fit index (AGFI), root mean square error of approximation (RMSEA) and Akaike information criterion (AIC). GFI and AGFI are used to measure how well the model fits the data, which varies from 0 to 1, with .90 indicating an acceptable model fit and 0.95 indicating a good model fit $[32,33]$. RMSEA is a measure of the discrepancy between a population-based model and a hypothesized model assessed per degree of freedom. There is good model fit if the RMSEA is less than or equal to 0.05 , with the upper limit of confidence interval less than 0.08 and the lower $90 \%$ confidence limit including or close to 0 [33]. A lower AIC value for a model reflects a better-fitting model compared with competing models [34]. A model was considered to fit the data when the following criteria were met: GFI > 0.90, AGFI $>0.90$ (AGFI $<$ GFI), RMSEA $<0.05$ and a lower AIC value than competing models. A p value less than 0.05 was considered statistically significant.

To adjust the original specified model, new free parameters were added based on the modified indices before the Wald test that deleted all non-significant free parameters to increase model fitness. Then only significant causal paths with corresponding standardized regression coefficients $(\beta)$ were shown in the figures of final structural models that demonstrated a good model fit. With the standardized regression coefficients, the magnitude of each factor could be directly compared with other factors in the model.

\section{RESULTS}

\subsection{Participant Characteristics}

Adolescent girls (mean age $=13.44, \mathrm{SD}=0.93$ ) with complete data comprised the final sample. Mean height and weight were $155.37 \mathrm{~cm}(\mathrm{SD}=5.32)$ and $46.98 \mathrm{~kg}$ $(\mathrm{SD}=8.72)$, respectively. The majority of adolescents had normal weight $(5 \leq$ BMI $<85$ percentile, $\mathrm{n}=236$, $84.3 \%$ ). More information about characteristics of the studied variables is provided in Table 1.

\subsection{Structural Equation Model for Lunch-Time Physical Activity}

The final structural model for lunchtime physical activity in Figure 1 demonstrated a good model fit (GFI = 0.95, AGFI $=0.93$, RMSEA $=0.02[90 \%$ confidence interval $=0.00-0.04]$ ). The recalculation of the model after addition and deletion of free parameters reduced the AIC value from 780.95 to 215.56 . During lunch recess, perceived friend support $(\beta=0.11)$ was found to have a direct positive effect on girls' physical activity. Self- 
Table 1. Characteristics of participants and outcome physical activity variables.

\begin{tabular}{lcc}
\hline & \multicolumn{2}{c}{ Participants $\left(\mathrm{N}^{\mathrm{a}}=280\right)$} \\
\cline { 2 - 3 } & Mean & $\mathrm{SD}^{\mathrm{a}}$ \\
\hline Age & 13.44 & 0.93 \\
Height & 155.37 & 5.32 \\
Weight & 46.98 & 8.72 \\
BMI & 19.40 & 3.14 \\
Lunch-time Physical Activity & 8.46 & 26.74 \\
After-school Physical Activity & 138.89 & 259.05 \\
Grade (N, \%) & & \\
$\quad$ Grade 1 & 99 & $35.4 \%$ \\
$\quad$ Grade 2 & 96 & $34.3 \%$ \\
$\quad$ Grade 3 & 85 & $30.4 \%$ \\
Weight status (N, \%) & & \\
$\quad$ Underweight & 12 & $4.3 \%$ \\
Normalweight & 236 & $84.3 \%$ \\
Overweight & 22 & $7.9 \%$ \\
$\quad$ Obesity & 10 & $3.6 \%$ \\
\hline
\end{tabular}

${ }^{a} \mathrm{~N}$ : number; SD: standard deviation.

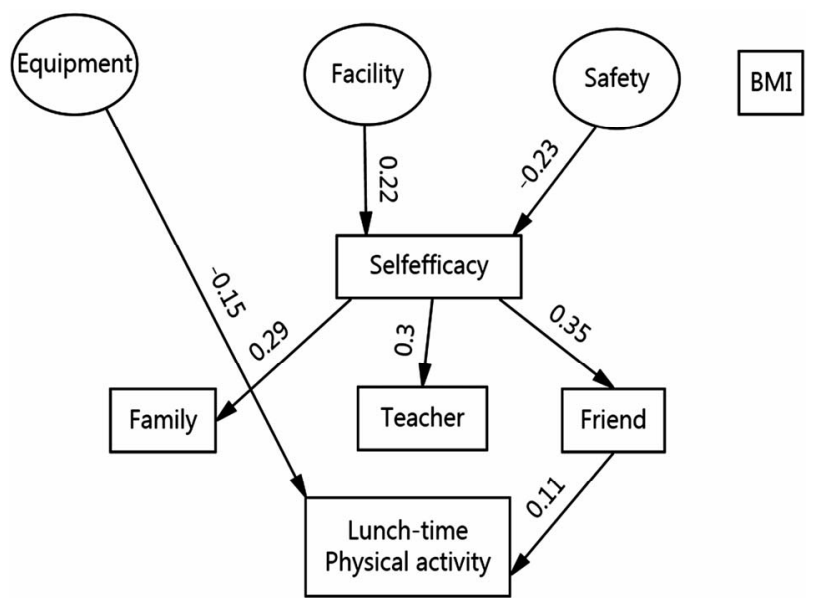

Figure 1. Effects of personal, social and environmental factors on lunch-time physical activity. Only statistically significant paths are shown in the figure. The significance level was set at $\mathrm{p}<0.05$. BMI: body mass index; Family: family support; Teacher: teacher support; Friend: friend support.

efficacy $(\beta=0.04)$ indirectly influenced physical activity through friend support. With respect to the influences of school environmental factors, perceived equipment exhibited a direct negative effect $(\beta=-0.15)$ on physical activity. The total effects of perceived facilities $(\beta=0.01)$ and safety $(\beta=-0.01)$ on physical activity were fully mediated by self-efficacy and friend support. Equipment was identified as the most influential environmental factor related to physical activity. There were no significant associations of BMI, family support or teacher support with physical activity.

\subsection{Structural Equation Model for After-School Physical Activity}

The final structural equation model for after-school physical activity in Figure 2 also demonstrated a good model fit $(\mathrm{GFI}=0.95$, AGFI $=0.93$, RMSEA $=0.03$ $[90 \%$ confidence interval $=0.00-0.04]$ ). The AIC value was reduced from 842.24 to 219.74 after the model modifications. In the final structural model, perceived equipment, teacher support and BMI failed to exhibit direct or indirect effects on physical activity. Perceived facilities $(\beta=0.02)$ and safety $(\beta=-0.02)$ were found to indirectly affect physical activity through self-efficacy and family support or friend support. Their effect sizes on physical activity were generally low. The standardized indirect effect of self-efficacy on physical activity through family and friend support was 0.09. Support from friends $(\beta=0.16)$ and family $(\beta=0.13)$ were found to directly affect physical activity. The final model identified friend support as the most influential factor directly affecting physical activity during after-school hours.

\section{DISCUSSION}

The present cross-sectional study examined effects of perceived school physical environment, social support, self-efficacy and BMI on school-based physical activity in adolescent girls.

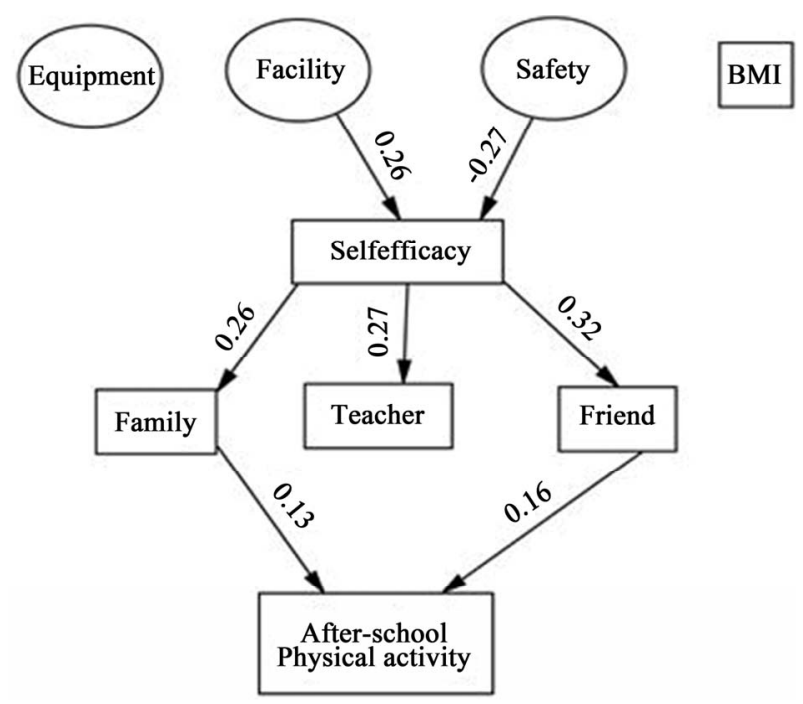

Figure 2. Effects of personal, social and environmental factors on after-school physical activity. Only statistically significant paths are shown in the figure. The significance level was set at $\mathrm{p}<0.05$. BMI: body mass index; Family: family support; Teacher: teacher support; Friend: friend support. 
The primary finding of this study was that social support directly affected school-based physical activity as a possible mediator. Specifically, friend support was found to be the source of support most highly related to physical activity in both lunchtime and after-school settings. Previous studies have demonstrated similar findings on moderate-to-vigorous physical activity during nonschool-time and mean daily physical activity in adolescent girls [11,35-38]. Collectively, findings suggest that developing strategies to encourage or assist with friends' physical activity behaviors can be beneficial in promoting physical activity in adolescent girls, regardless of contexts. Compared with friend support, support from family and teachers for physical activity were found to be less important in both contexts. This finding is understandable because adolescent girls are becoming independent from family and strengthening their identification with peers $[11,37]$. Interestingly, the effect of family support on physical activity was significant in the after-school context but not at lunch time. This suggests that parents mainly act as a "gate keeper" by providing instrumental support (e.g., transportation and providing access to equipment) so that children can engage in organized physical activity during after-school hours [39, 40]. Similarly, the non-significant association of teacher support with physical activity was understandable because physical activity becomes a free choice in leisure time. Teachers' influence may be more significant in physical education courses rather than in free time behavioral choices. In Japan, approximately $71.2 \%$ of junior high school girls join in their school's extracurricular sports clubs during after-school hours. Only $0.7 \%$ of girls are motivated by teachers. However, $26.4 \%$ of report that they take part in extracurricular sports clubs because of their friends and family [41]. Considering that no comparable previous studies exist, to better understand the context-specific difference in social support related to physical activity, more in-depth research is needed to examine preferred types of physical activity in different contexts, and to identify types of support (e.g., encouragement or tangible assistance) in association with context-specific physical activity. Findings in thepresent study suggest considering family and friend support as possible mediators in future longitudinal or intervention studies to observe changes of physical activity in specific contexts. Based on available evidence $[35,42]$, when testing the interventional effects of social support on direct promotion of physical activity at school, the following should be considered: increasing the frequency of parents or friends watching or talking about physical activity, parents or friends increasing their encouragement of girls joining in extracurricular sport clubs started at school and parental assistance with fees for equipment needed for these clubs.
Self-efficacy has been demonstrated as a consistent correlate and mediator of adolescents' physical activity $[10,11,14,16,43,44]$. However, in the current study, selfefficacy did not directly affect physical activity of adolescent girls. The inconsistency between previous studies and the current study might be attributed to aspects of self-efficacy measured. The present study focused on the self-efficacy in performance of activities; previous studies primarily examined barriers self-efficacy. Ryan et al. found that the impacts of different types of self-efficacy (e.g., barriers self-efficacy, performance self-efficacy and asking self-efficacy) on physical activity were different [30]. Additionally, our finding might further confirm the study of Dishman et al. suggesting that physical activity interventions designed to enhance self-efficacy might be especially needed during preadolescence [7]. Therefore, to gain a complete understanding of the relationship between self-efficacy and context- specific physical activity, future studies should include more aspects of self-efficacy that may directly account for the variance in physical activity, and should follow changes in self-efficacy throughout primary and junior high school.

This study provided additional evidence of associations between different features of school physical environment and physical activity by context [45-48]. First, perceived equipment had a direct negative effect on girls' lunchtime physical activity. It is possible that girls who actually used equipment reported a lower score for available equipment than girls who did not use it because the latter were less aware of the real equipment situation. Second, the negative effect of perceived safety on school-based physical activity might be accounted for by inaccurate measures of the physical environment. Some previous studies have shown that the agreement between perceived and objectively measured environment is often poor, and the relationship between objective and self-report measures of physical environment and physical activity is inconsistent among adolescents $[49,50]$. Therefore, future research is needed to test whether perceptions of equipment and safety match objective measurements to clarify the influences of equipment and safety on physical activity. In addition, this study indicated that increasing perceptions of accessible facilities at school might promote physical activity participation among adolescent girls through increasing self-efficacy and social support. This information highlights the importance of increasing information channels regarding available or accessible facilities at school among students or parents. There are various ways to accomplish this. For instance, school officials could report to students and parents in timely manner updated news about regular maintenance of facilities, or install a sign on school grounds informing students about the 
available facilities and policies about using them.

One more finding of this study was that BMI had no significant influence on girls' physical activity. BMI is one of the most studied biological markers of body weight and shape. Evidence from systematic reviews have shown that there is no consistent association between BMI and adolescent girls' physical activity level, with the majority of studies reporting either a small negative or no correlation $[43,51,52]$. The small or nonsignificant effects of BMI may suggest that other potentially body weight-and shape-related factors like body image need to be assessed in future behavioral models for girls. Different from BMI, the construct of body image measured physical appearance attitudinally, consisting of subjective feelings and beliefs on one's own appearance (e.g., body dissatisfaction) [53] and perceptions of how the body moves and functions, or what the body can "do" [54]. Considering cultural influences and norms on poor and ideal body image and that girls tend to be more concerned about their physical appearance than boys during adolescence, body image rather than BMI is often thought to be more likely to reflect variances of physical activity or other weight-related behavior in adolescent girls [43,55]. Therefore, it would be worthwhile including the construct of body image in future models.

The collective evidence is sufficient to encourage the use of support from friends and families as mediators in future intervention studies designed to increase physical activity among Japanese adolescent girls. Given that differences between school systems and environments may limit the generalizability of findings between countries, and many previous studies were conducted in Western countries, research on Japanese adolescent girls is needed. There are several strengths of the present research worth noting. First, this study extended previous research by measuring both direct and indirect effects of multilevel contributing factors on context-specific physical activity rather than overall physical activity level. In addition, several sources of social support and various school physical environmental attributes were examined concurrently in the present study. Although previous studies have compared the effects of family and friend support on physical activity, there are no studies that simultaneously investigated the relative importance of family, friends and teachers on girls' school-based physical activity. Second, the present study contributed to studies on adolescents by exploring the mechanisms underlying the physical activity behavior of Asian adolescent girls. Finally, the present study used SEM analysis which was helpful in exploring potential mediators that can possibly intervene and allowed examination of relative contributions of factors explaining physical activity.
Despite the strengths, a number of limitations must be noted. One is the use of a self-report measure of physical activity, which is subject to error and bias. Further studies should combine existing objective and subjective measures to investigate context-specific physical activity more accurately. Furthermore, lack of objective measurement of the physical environment characteristics may account for the inaccurate perceptions of the physical environment. Future research should use both self-report and objective measures to accurately evaluate the environment. Another limitation is the list-wise deletion adopted in the present study, which may lead to bias in data findings. A further limitation is that the generalizebility of findings beyond the study location may be limited by the data from a single school. However, the prevalence of overweight and obesity in the present study $(11.5 \%)$ was slightly higher than the prevalence in a national survey $(7.4 \%)$ [22]. This implies that the structural models in the present study might be reflective of counterparts across the country. Still another limitation is the cross-sectional data used in this study, which only permits estimates of between-person relations among variables. Therefore, a longitudinal or interventional design that permits estimates of the effects of changes of mediators on the physical activity within participants is warranted in future research.

In summary, the present study indicated that 1) social support mediated the cross-sectional effect of physical environment and self-efficacy on physical activity among adolescent girls and 2) associations between examined factors and school-based physical activity differed by context. Results imply that it is possible to develop a school-based intervention through collective supports from families and friends to increase girls' school-based physical activity level. Furthermore, the mediated effect of perceptions of self-efficacy and physical environment that may be helpful for increasing perceptions of social support should be tested in future studies.

\section{ACKNOWLEDGEMENTS}

The authors would like to thank the participating students and school in the present study. This investigation was supported by the Grantsin-Aid for Scientific Research (No. 22700680) from the Japan Society for the Promotion of Science, Waseda University Grant for Special Research Projects (2010A-095, 2011A-092), and the Global COE Program "Sport Sciences for the Promotion of Active Life" from the Japan Ministry of Education, Culture, Sports, Science, and Technology.

\section{REFERENCES}

[1] Hallal, P.C., Victora, C.G., Azevedo, M.R. and Wells, J.C. (2006) Adolescent physical activity and health: A systematic review. Sports Medicine, 36, 1019-1030. doi:10.2165/00007256-200636120-00003 
[2] Janssen, I. and LeBlanc, A.G. (2010) Systematic review of the health benefits of physical activity and fitness in school-aged children and youth. International Journal of Behavioral Nutrition and Physical Activity, 7, 40. doi:10.1186/1479-5868-7-40

[3] World Health Organization (2012) Global recommendation on physical activity for health. http://whqlibdoc.who.int/publications/2010/97892415999 79 eng.pdf

[4] Ministry of Education, Culture, Sports, Science and Technology, Japan (2012) 2010's national survey result of physical and athletic capacity and exercise habits: (6) sports and exercise habits.

http:/www.mext.go.jp/component/a_menu/sports/detail/ icsFiles/afieldfile/2010/12/16/1300270 061 1.pdf

[5] Baron, R. and Kenny, D. (1986) The moderator-mediator variable distinction in social psychological research: conceptual, strategic, and statistical considerations. Journal of Personality and Social Psychology, 51, 1173-1182. doi:10.1037/0022-3514.51.6.1173

[6] Dishman, R.K., Motl, R.W., Saunders, R., Felton, G., Ward, D.S. and Dowda, M., et al. (2004) Self-efficacy partially mediates the effect of a school-based physical activity intervention among adolescent girls. Preventive Medicine, 38, 628-636. doi:10.1016/j.ypmed.2003.12.007

[7] Dishman, R.K., Saunders, R.P., Motl, R.W., Dowda, M., and Pate, R.R. (2009) Self-efficacy moderates the relation between declines in physical activity and perceived social support in high school girls. Journal of Pediatric Psychology, 34, 441-451. doi:10.1093/jpepsy/jsn100

[8] Dishman, R.K., Dunn, A.L., Sallis, J.F., Vandenberg, R.J. and Pratt, C.A. (2010) Social-cognitive correlates of physical activity in a multi-ethnic cohort of middleschool girls: Two-year prospective study. Journal of Pediatric Psychology, 35, 188-198. doi:10.1093/jpepsy/jsp042

[9] Hsu, YW, Chou, C.P., Nguyen-Rodriguez, S.T., McClain, A.D., Belcher, B.R. and Spruijt-Metz, D. (2011) Influences of social support, perceived barriers, and negative meanings of physical activity on physical activity in middle school students. Journal of Physical Activity and Health, 8, 210-219.

[10] Trost, S.G., Sallis, J.F., Pate, R.R., Freedson, P.S., Taylor, W.C. and Dowda, M. (2003) Evaluating a model of parental influence on youth physical activity. American Journal of Preventive Medicine, 25, 277-282. doi:10.1016/S0749-3797(03)00217-4

[11] Wu, T., Pender, N. and Noureddine, S. (2003) Gender differences in the psychosocial and cognitive correlates of physical activity among Taiwanese adolescents: A structural equation modeling approach. International Journal of Behavioral Medicine, 10, 93-105. doi:10.1207/S15327558IJBM1002 01

[12] Motl, R.W., Dishman, R.K., Ward, D.S., Saunders, R.P., Dowda, M. and Felton, G., et al. (2005) Comparison of barriers self-efficacy and perceived behavioral control for explaining physical activity across 1 year among adolescent girls. Health Psychology, 24, 106-111. doi:10.1037/0278-6133.24.1.106
[13] Motl, R.W., Dishman, R.K., Ward, D.S., Saunders, R.P., Dowda, M. and Felton, G., et al. (2005) Perceived physical environment and physical activity across one year among adolescent girls: Self-efficacy as a possible mediator? Journal of Adolescent Health, 37, 403-408. doi:10.1016/j.jadohealth.2004.10.004

[14] Motl, R.W., Dishman, R.K., Saunders, R.P., Dowda, M. and Pate, R.R. (2007) Perceptions of physical and social environment variables and self-efficacy as correlates of self-reported physical activity among adolescent girls. Journal of Pediatric Psychology, 32, 6-12. doi:10.1093/jpepsy/js1001

[15] Lubans, D.R., Okely, A.D., Morgan, P.J., Cotton, W., Puglisi, L. and Miller, J. (2012) Description and evaluation of a social cognitive model of physical activity behaviour tailored for adolescent girls. Health Education Research, 27, 115-128. doi:10.1093/her/cyr039

[16] Van der Horst, K., Paw, M.J., Twisk, J.W. and Van Mechelen, W. (2007) A brief review on correlates of physical activity and sedentariness in youth. Medicine \& Science in Sports \& Exercise, 39, 1241-1250. doi:10.1249/mss.0b013e318059bf35

[17] McKenzie, T.L., Marshall, S.J., Sallis, J.F. and Conway, T.L. (2000) Leisure-time physical activity in school environments: An observational study using SOPLAY. Preventive Medicine, 30, 70-77. doi:10.1006/pmed.1999.0591

[18] Ridgers, N., Stratton, G. and Fairclough, S. (2006) Physical activity levels of children during school playtime. Sports Medicine, 36, 359-371. doi:10.2165/00007256-200636040-00005

[19] Ministry of Health, Labour, and Welfare, Japan (2007) 2007's national survey results of Japanese health and nutrition status.

http://www.mhlw.go.jp/bunya/kenkou/eiyou09/dl/01-03.p df

[20] Ministry of Health, Labour, and Welfare, Japan (2008) 2008 's national survey results of Japanese health and nutrition status.

http://www.mhlw.go.jp/bunya/kenkou/eiyou/dl/h20-houk oku-03.pdf

[21] Ministry of Education, Culture, Sports, Science and Technology, Japan (2009) 2009's national survey result of physical and athletic capacity and exercise habits: Physical fitness and BMI.

http://www.mext.go.jp/component/a menu/sports/detail/ icsFiles/afieldfile/2009/12/17/1287809 4.pdf

[22] Ministry of Education, Culture, Sports, Science and Technology, Japan (2010) 2010's national survey result of physical and athletic capacity and exercise habits: Physical fitness and BMI.

http://www.mext.go.jp/component/a_menu/sports/detail/ _icsFiles/afieldfile/2010/12/16/1300270_4_1.pdf

[23] Sano, A., Le, D.S., Tran, M.H., Pham, H.T., Kaneda, M. and Murai E., et al. (2008) Study on factors of body image in Japanese and Vietnamese adolescents. Journal of Nutritional Science and Vitaminology, 54, 169-175. doi:10.3177/jnsv.54.169

[24] Berk, L.E. (2005) Infants, children and adolescents. 5th 
Edition, Allyn \& Bacon Inc., Bosten.

[25] Koizumi K. (1997) Adaptation process during transition from elementary school to junior high school. (Shōchūgakkō de no kankyō ikō jitai ni okeru jidō, seito no tekiō katei chūgakkō nyūgaku, tenkō o chūshin to shite, in Japanese). Kasama Shobo, Tokyo.

[26] Cole, T., Bellizzi, M., Flegal, K. and Dietz, W. (2000) Establishing a standard definition for child overweight and obesity worldwide: International survey. Brtish Medical Journal, 320, 1240-1243. doi:10.1136/bmj.320.7244.1240

[27] Centers for Disease Control and Prevention (2011) About BMI for children and teens. http://www.cdc.gov/healthyweight/assessing/bmi/children s_bmi/about_childrens bmi.html

[28] He, L., Ishii, K., Shibata, A., Adachi, M., Nonoue, K. and Oka K. (2012) Patterns of physical activity outside of school time among Japanese junior high school students. Journal of School Health, in Press.

[29] Robert-Wilson, J., Levesque, L. and Holden, R.R. (2007) Development of a questionnaire assessing school physical activity environment. Measurement in Physical Education and Exercise Science, 11, 93-107. doi:10.1080/10913670701294088

[30] Ryan, G. and Dzewaltowski, D. (2002) Comparing the relationships between different types of self-efficacy and physical activity in youth. Health Education \& Behavior, 29, 491-504. doi:10.1177/109019810202900408

[31] North Carolina State University (2012) Structural equation modeling: Statnotes. North Carolina State University, Raleigh. http://faculty.chass.ncsu.edu/garson/PA765/structur.htm

[32] Kline, R.B. (1998) Principle and practice of structural equation modeling. Guilford, New York.

[33] Schumacker, R.E. and Lomax, R.G. (2004) A beginner's guide to structural equation modeling. 2nd Edition, Lawrence Erlbaum Associates, Mahwah.

[34] Hamparsum, B. (1987) Model selection and Akaike's information criterion (AIC): The general theory and its analytical extensions. Psychometrika, 52, 345-370. doi:10.1007/BF02294361

[35] Duncan, S., Duncan, T. and Strycker, L. (2005) Sources and types of social support in youth physical activity. Health Psychology, 24, 3-10. doi:10.1037/0278-6133.24.1.3

[36] Hohepa, M., Scragg, R., Schofield, G., Kolt, G.S. and Schaaf, D. (2007) Social support for youth physical activity: Importance of siblings, parents, friends and school support across a segmented school day. International Journal of Behavioral Nutrition and Physical Activity, 4, 54. doi:10.1186/1479-5868-4-54

[37] Lytle, L.A., Murray, D.M., Evenson, K.R., Lytle, L.A., Murray, D.M. and Evenson, K.R., et al. (2009) Mediators affecting girls' levels of physical activity outside of school: Findings from the trial of activity in adolescent girls. Annals of Behavioral Medicine, 38, 124-136. doi:10.1007/s12160-009-9127-2

[38] Patnode, C.D., Lytle, L.A., Erickson, D.J., Sirard, J.R.,
Barr-Anderson, D. and Story, M. (2010) The relative influence of demographic, individual, social, and environmental factors on physical activity among boys and girls. International Journal of Behavioral Nutrition and Physical Activity, 7, 79. doi:10.1186/1479-5868-7-79

[39] Alderman, B.L., Benham-Deal, T.B. and Jenkins, J.M. (2010) Change in parental influence on children's physical activity over time. Journal of Physical Activity and Health, 7, 60-67.

[40] Welk, G., Wood, K. and Morss, G. (2003) Parental influences on physical activity in children: An exploration of potential mechanisms. Pediatric Exercise Science, 15, 19-33.

[41] Ministry of Education, Culture, Sports, Science and Technology, Japan (2010) 2010's national survey result of physical and athletic capacity and exercise habits. http://www.mext.go.jp/component/a_menu/sports/detail/ icsFiles/afieldfile/2010/12/16/1300103 5.pdf

[42] Robbins, L.B., Stommel, M. and Hamel, L.M. (2008) Social support for physical activity of middle school students. Public Health Nursing, 25, 451-460. doi:10.1111/j.1525-1446.2008.00729.x

[43] Biddle, S.J.H., Whitehead, S.H.O., Donovan, T.M. and Nevill, M.E. (2005) Correlates of participation in physical activity for adolescent girls: A systematic review of recent literature. Journal of Physical Activity and Health, 2, 423-434.

[44] Salmon, J., Brown, H. and Hume, C. (2009) Effects of strategies to promote children's physical activity on potential mediators. International Journal of Obesity, 33, S66-S73. doi:10.1038/ijo.2009.21

[45] Haug E., Torsheim T. and Samdal O. (2008) Physical environmental characteristics and individual interests as correlates of physical activity in Norwegian secondary schools: The health behaviour in school-aged children study. International Journal of Behavioral Nutrition and Physical Activity, 5, 47. doi:10.1186/1479-5868-5-47

[46] Haug, E., Torsheim, T., Sallis, J.F. and Samdal, O. (2010) The characteristics of the outdoor school environment associated with physical activity. Health Education Research, 25, 248-256. doi:10.1093/her/cyn050

[47] Prins, R.G., van Empelen, P., Velde, S.J.T., Timperio, A., van Lenthe, F.J. and Tak N.I., et al. (2010) Availability of sports facilities as moderator of the intention-sports participation relationship among adolescents. Health Education Research, 25, 489-497. doi:10.1093/her/cyq024

[48] Colabianchi, N., Maslow, A.L. and Swayampakala, K. (2011) Features and amenities of school playgrounds: A direct observation study of utilization and physical activity levels outside of school time. International Journal of Behavioral Nutrition and Physical Activity, 8, 32

[49] Maddison, R., Jiang, Y., Hoorn, S.V., Mhurchu, C.N., Exeter, D. and Utter J. (2010) Perceived versus actual distance to local physical-activity facilities: Does it really matter? Journal of Physical Activity and Health, 7, 323332.

[50] McCormack, G., Giles-Corti, B., Lange, A., Smith, T., 
Martin, K. and Pikora, T.J. (2004) An update of recent evidence of the relationship between objective and selfreport measures of the physical environment and physical activity behaviors. Journal of Science and Medicine in Sport, 7, 81-92. doi:10.1016/S1440-2440(04)80282-2

[51] Sallis J., Prochaska J. and Taylor W. (2000) A review of correlates of physical activity of children and adolescents. Medicine \& Science in Sports \& Exercise, 32, 963-975. doi:10.1097/00005768-200005000-00014

[52] Van der Horst, K., Paw, M.J.C.A., Twisk, J.W.R. and Van Mechelen, W. (2007) A brief review on correlates of physical activity and sedentariness in youth. Medicine \& Science in Sports \& Exercise, 39, 1241-1250. doi:10.1249/mss.0b013e318059bf35

[53] Thompson, J.K., Heinberg, L.J., Altabe, M.N. and Tantleff-
Dunn, S. (1999) Exacting beauty: Theory, assessment, and treatment of body image disturbance. American Psychological Association, Washington DC. doi:10.1037/10312-000

[54] Abbott, B.D. and Barber, B.L. (2011) Differences in functional and aesthetic body image between sedentary girls and girls involved in sports and physical activity: Does Sport Type Make a Difference? Psychology of Sport and Exercise, 12, 333-342. doi:10.1016/j.psychsport.2010.10.005

[55] Rauste-von Wright, M. (1989) Body image satisfaction in adolescent girls and boys: A longitudinal study. Journal of Youth and Adolescence, 18, 71-83. doi:10.1007/BF02139247 